\section{(6) OPEN ACCESS}

\title{
Physical activity at age 11 years and chronic disabling fatigue at ages 13 and 16 years in a UK birth cohort
}

\author{
Simon M Collin, ${ }_{1}^{1,2}$ Tom Norris, ${ }^{3}$ Kevin C Deere, ${ }^{4}$ Russell Jago, ${ }^{5}$ Andy R Ness, ${ }^{6}$ \\ Esther Crawley ${ }^{1,2}$
}

\begin{abstract}
- Additional material is published online only. To view please visit the journal online (http://dx.doi.org/10.1136/ archdischild-2017-314138)
\end{abstract}

${ }^{1}$ Population Health Sciences, Bristol Medical School, University of Bristol, Bristol, UK ${ }^{2}$ Centre for Child and Adolescent Health, University of Bristol, Bristol, UK

${ }^{3}$ Department of Health Sciences, Centre for Medicine, College of Medicine, Biological Sciences and Psychology, University of Leicester, Leicester, UK

${ }^{4}$ Musculoskeletal Research Unit, University of Bristol, Southmead Hospital, Bristol, UK

${ }^{5}$ Centre for Exercise, Nutrition and Health Sciences, School for Policy Studies, University of Bristol, Bristol, UK

${ }^{6} \mathrm{NIHR}$ Biomedical Research Centre at the University Hospitals Bristol NHS Foundation Trust and the University of Bristol, University Hospitals Bristol Education Centre, Bristol, UK

\section{Correspondence to} Professor Esther Crawley, Centre for Child and Adolescent Health, Population Health Sciences, Bristol Medical School University of Bristol, Bristol BS8 2BN, UK;

esther.crawley@bristol.ac.uk

Received 19 September 2017 Revised 6 January 2018 Accepted 10 January 2018 Published Online First 30 January 2018

Check for updates

To cite: Collin SM, Norris T, Deere KC, et al.

Arch Dis Child

2018:103:586-591.

\section{ABSTRACT}

Objective To investigate associations of physical activity at age 11 years with chronic disabling fatigue (CDF) at ages 13 and 16 years.

Design Longitudinal birth cohort.

Setting South-West England.

Participants Adolescents enrolled in the Avon Longitudinal Study of Parents and Children.

Outcomes and exposures We identified adolescents who had disabling fatigue of $>6$ months' duration without a known cause at ages 13 and 16 years. Total and moderate-to-vigorous physical activity and sedentary time at age 11 years were measured by accelerometry over a 7-day period.

Results A total physical activity level 100 counts/min higher at age 11 years was associated with 25\% lower odds of CDF at age 13 years (OR=0.75 (95\% Cl 0.59 to 0.95$))$, a $1 \%$ increase in the proportion of monitored time spent in moderate-to-vigorous activity was associated with $16 \%$ lower odds of CDF (OR $=0.84$ (95\% $\mathrm{Cl} 0.69$ to 1.01)) and a 1-hour increase in sedentary time was associated with $35 \%$ higher odds of CDF $(\mathrm{OR}=1.35(95 \% \mathrm{Cl} 1.02$ to 1.79$))$. Disabling fatigue of only 3-5 months' duration at age 13 years had weaker associations with physical activity, and CDF at age 16 years was not associated with physical activity at age 11 years.

Conclusions Children who had chronic disabling fatigue at age 13 years had lower levels of total and moderate-to-vigorous physical activity and more sedentary time 2 years previously, but this association could be explained by reverse causation.

\section{INTRODUCTION}

Chronic fatigue syndrome (CFS), also known as 'ME' and, in the USA, as systemic exertion intolerance disease, ${ }^{1}$ is a debilitating disease which affects adults and children. ${ }^{2}{ }^{3}$ UK National Institute for Health and Care Excellence guidelines state that a diagnosis of CFS/ME should be made after 3 months of persistent or recurrent fatigue which is not the result of ongoing exertion, not alleviated by rest, has resulted in a substantial reduction in activities, has no other known cause and is accompanied by one or more of 10 typical symptoms. ${ }^{4}$ The US Centers for Disease Control and Prevention diagnostic criteria are similar, but specify 6 months' duration of fatigue and four or more additional symptoms. ${ }^{5}$ Chronic fatigue of at least 3 months' duration affects $1 \%-3 \%$ of children and young people. $^{6-11}$ Clinician-verified CFS/ME appears to be much less prevalent $(0.1 \%-0.5 \%)^{12-14}$; this

\section{What is already known on this topic?}

- Lower levels of childhood physical activity were reported to be associated with increased risk of chronic fatigue syndrome (also known as ME) during adulthood.

- It is unclear whether physical activity is a risk factor for the development of chronic fatigue during adolescence.

\section{What this study adds?}

- Children who were less physically active and more sedentary at age 11 years had an increased risk of chronic disabling fatigue at 13 years.

- These associations were not evident at age 16 years, and could be explained by reverse causation.

discrepancy is probably a combination of clinician diagnostic uncertainty, ${ }^{15}$ undiagnosed cases ${ }^{16}$ and inaccuracies in population-based studies.

It is unclear whether physical activity is a risk factor for paediatric CFS/ME. Lower levels of childhood physical activity were reported to be associated with increased risk of $\mathrm{CFS} / \mathrm{ME}$ during adulthood, ${ }^{17}$ and healthy girls aged 14 years girls who were less physically active were more likely to report symptoms characteristic of CFS/ME (unrefreshing sleep, muscle pain, joint pain, headaches, tender lymph nodes, memory and concentration problems) at age 19 years. ${ }^{18}$ A case-control study of adolescents with infectious mononucleosis who did (cases) or did not (controls) subsequently develop CFS/ME found no differences in levels of physical activity in the 12 months before the initial infectious illness. ${ }^{19}$ Here we use data from the Avon Longitudinal Study of Parents and Children (ALSPAC) to investigate whether levels and patterns of physical activity at age 11 years $^{20}$ are associated with 'chronic disabling fatigue' (CDF, a proxy for clinically diagnosed CFS/ $\mathrm{ME})$ at ages 13 and 16 years. $^{67}$

\section{METHODS}

Study cohort

ALSPAC is a population-based study that aims to investigate a wide range of influences on the health and development of children. ${ }^{21}$ Pregnant women residing in the former Avon Health Authority in 
South-West England who had an estimated date of delivery between 1 April 1991 and 31 December 1992 were invited to take part, resulting in a cohort of 14541 pregnancies and 13978 children alive at 12 months of age (excluding triplets and quads). The ALSPAC study website contains details of all the data that are available through a fully searchable data dictionary (www. bris.ac.uk/alspac/researchers/data-access/data-dictionary/).

\section{Outcomes-CDF at ages 13 and 16 years}

Our methods for defining CDF at age 13 (median 13.1, IQR 13.1-13.2) and 16 (median 16.6, IQR 16.5-16.8) years have been described previously. ${ }^{6}$ In brief, mothers were asked whether their child had been feeling tired or lacking in energy over the last month (yes, no); how long the tiredness had lasted ( $<3$ months, $3-5$ months, 6 months to 5 years, $>5$ years); how many days their child had missed school because of tiredness; and whether the tiredness/lack of energy had prevented the child from taking part in hobbies, sport or leisure activities (not at all, only a little, quite a lot, a great deal). The questionnaires asked whether the teenager snored (never, sometimes, often), whether the mother thought the fatigue was due to the teenager playing too much sport and whether the teenager took regular medication (free text question: 'Please indicate below any medicines your child has used in the last 12 months'). We identified adolescents reported by their mothers to have experienced fatigue lasting $>6$ months that was associated with absence from full-time school or that had prevented them from taking part in activities 'quite a lot' or 'a great deal'. We excluded those whose mothers thought that the fatigue was caused by playing too much sport, who snored often and who had other illnesses that could cause fatigue (based on self-reported medication use). At age 16 years, children could only be classified as having CDF if they scored $\geq 19$ (out of 33) on the Chalder Fatigue Scale. $^{22} 23$ According to our definitions, the prevalence of CDF of $>6$ months' duration at ages 13 and 16 years was $1.1 \%(76 / 6720)$ and $1.5 \%(84 / 5756)$, respectively. To reduce overlap between primary exposures and outcomes, we excluded children with $>5$ years' duration of fatigue at ages 13 (10 out of 76 children) and 16 (11 out of 84 children) years. After these exclusions, 66/6720 children at age 13 years and 73/5756 children at age 16 years were classified as having CDF (of 6 months' to 5 years' duration). We also performed a sensitivity analysis in which we redefined our outcome at age 13 years to include only children whose disabling fatigue had been of 3-5 months' duration $(78 / 6720(1.2 \%))$.

\section{Primary exposure-physical activity at age 11 years}

Methods for collecting physical activity data have been described previously. ${ }^{20}$ All ALSPAC children who attended research clinics at age 11 years (median 11.8 years, IQR 11.6-11.9 years) were asked to wear an Actigraph AM7164 2.2 accelerometer (Actigraph LLC, Fort Walton Beach, Florida, USA) for 7 days. Data are recorded as counts, which are averaged over a defined period ( 1 min in this study). The Actigraph has been validated in children and adolescents. ${ }^{24-27}$ Participants wore the accelerometer on a waist worn belt and were instructed to remove it only when showering, bathing or doing water sports. Physical activity variables were derived from the raw accelerometer counts using customised software. Data from children who had worn the accelerometer for at least 10 hours a day for at least 3 days were considered valid. Three physical activity variables were calculated-total (volume of) physical activity, moderate-to-vigorous physical activity and sedentary time.
Total physical activity was calculated as the average accelerometer counts per minute (cpm) over the full period of valid recording. To facilitate interpretation, associations with total physical activity were calculated per $100 \mathrm{cpm}$. This measure was chosen because it approximates the difference in physical activity between boys and girls and therefore provides a useful reference point. ${ }^{20}$ All minutes of recording with a total of $\geq 3500$ accelerometer counts were classified as moderate-to-vigorous physical activity. The threshold of $3500 \mathrm{cpm}$ was derived from a calibration study in a subsample of 246 ALSPAC children. ${ }^{25}$ We analysed moderate-to-vigorous physical activity as a mean daily proportion of total monitored time. This allowed us to investigate whether the volume of physical activity or (occasional) intensity of physical activity was more important in predicting risk of CDF. Sedentary time was the mean daily amount of time (hours per day) when the accelerometer recorded $\leq 100 \mathrm{cpm}$.

\section{Statistical analysis}

A set of putative risk factors for paediatric CFS/ME was identified from the literature. ${ }^{671028}$ To identify possible associations between our exposures and outcome, we identified confounding variables which could potentially bias any observed association. ${ }^{29}$ The first step in this process was to hold consultative meetings with experts in the fields related to the primary (physical activity) and other exposures (sleep, child and maternal psychopathology) and specialists in paediatric CFS/ME. Consensus from these meetings was encapsulated in the form of directed acyclic graphs (DAGs). DAGs are causal diagrams which provide a method for formalising and clarifying relationships between variables, ${ }^{30}$ thereby informing the process of building causal models. ${ }^{31}$ DAGs are useful for identifying variables which confound the relationship between two variables, thus providing researchers with a set of variables for which adjustment is necessary (or unnecessary) in order to obtain unbiased estimates of the causal relationship between two variables. ${ }^{32}$ We refer to the final (logistic regression) model comprising the outcome (CDF as a binary variable), the primary exposure (total and/or moderate-to-vigorous physical activity as continuous variables) and all identified confounders as the 'analysis model' (online supplementary file figure S1-DAG generated by DAGitty software V.2.3, available from www.dagitty.net). ${ }^{33}$

If the analysis model is fitted to a 'complete case' dataset, that is, dropping participants who have missing data for any of the variables in the model, SEs will be inflated, and bias may arise. If missingness is dependent only on observed data, that is, data in the analysis model are 'missing at random', then multiple imputation can be used to correct this bias. Multiple imputation uses a model based on the analysis model plus auxiliary variables which are selected because (1) they are associated with the missing variables, (2) they are associated with the missingness of the missing variables and (3) they do not have too much missing data (to ensure stable imputation models which therefore produce reliable estimates).

The number of imputations required to achieve convergence of parameter estimates was determined by checking the estimate of the Monte Carlo error (MCE) in relation to the SE of the coefficient being estimated. The number of imputations was increased until MCE reached a value which was $<10 \%$ of the SE of the estimate. The sample size after imputation was $n=13978$, representing children in the 'core' ALSPAC sample who were alive at 1 year and who were either a singleton or twin. Multivariable imputation was performed using an imputation sampling method which incorporates all sources of variability 
Table 1 Physical activity at age 11 in children who did or did not have chronic disabling fatigue (CDF) at age 13 or 16 years

\begin{tabular}{|c|c|c|c|}
\hline & $\begin{array}{l}\text { Children without } \\
\text { CDF at age } 13 \text { years } \\
(n=4504)^{*}\end{array}$ & $\begin{array}{l}\text { Children with CDF at } \\
\text { age } 13 \text { years }(n=42)^{*}\end{array}$ & \\
\hline & Median (IQR) & Median (IQR) & $P$ valuet \\
\hline $\begin{array}{l}\text { Total physical activity } \\
\text { (counts/min) } \ddagger\end{array}$ & $577(472-710)$ & $482(409-581)$ & 0.001 \\
\hline $\begin{array}{l}\text { Moderate-to-vigorous } \\
\text { physical activity (\%)§ }\end{array}$ & $2.68(1.62-4.22)$ & $1.77(1.11-3.42)$ & 0.006 \\
\hline $\begin{array}{l}\text { Sedentary time } \\
\text { (hours)ף }\end{array}$ & $5.93(5.09-6.76)$ & $6.55(6.08-7.35)$ & 0.002 \\
\hline & $\begin{array}{l}\text { Children without } \\
\text { CDF at age 16years } \\
(n=3854)^{* *}\end{array}$ & $\begin{array}{l}\text { Children with CDF at } \\
\text { age } 16 \text { years }(n=53)^{* *}\end{array}$ & \\
\hline $\begin{array}{l}\text { Total physical activity } \\
\text { (counts/min) } \ddagger\end{array}$ & $573(468-705)$ & $526(432-667)$ & 0.08 \\
\hline $\begin{array}{l}\text { Moderate-to-vigorous } \\
\text { physical activity (\%)§ }\end{array}$ & $2.65(1.59-4.17)$ & $2.27(1.31-3.62)$ & 0.08 \\
\hline $\begin{array}{l}\text { Sedentary time } \\
\text { (hours)ף }\end{array}$ & $5.98(5.13-6.80)$ & $6.34(5.38-6.99)$ & 0.07 \\
\hline \multicolumn{4}{|c|}{$\begin{array}{l}\text { *Physical activity data at age } 11 \text { years were available for } 63.6 \%(42 / 66) \text { and } 67.7 \\
\text { (4504/6654) of children who did or did not have CDF at age } 13 \text { years, respectivel) } \\
\text { tKruskal-Wallis test. } \\
\text { ¥Total physical activity=mean counts/min over the } 7 \text {-day period when the } \\
\text { accelerometers were worn. } \\
\text { §Moderate-to-vigorous physical activity=percentage of monitored time when } \\
\text { accelerometer recorded } \geq 3500 \text { counts/min. } \\
\text { ISedentary time=mean daily time when accelerometer recorded } \leq 100 \\
\text { counts/min. } \\
\text { **Physical activity data at age } 11 \text { years were available for } 72.6 \%(53 / 73) \text { and } \\
67.8 \%(3854 / 5683) \text { of children who did or did not have CDF at age } 16 \text { years, } \\
\text { respectively. }\end{array}$} \\
\hline
\end{tabular}

and uncertainty in the imputed values. ${ }^{34}$ The analysis model is then fitted to the imputed datasets, using Rubin's rules to combine the estimates into a single estimate which is unbiased (or less biased) by differential losses to follow-up. ${ }^{35}$ We also used the imputed datasets to estimate (using linear regression) the mean differences in total and moderate-to-vigorous physical activity and sedentary time at age 11 years comparing children who did or did not develop CDF at age 13 or 16 years. This estimated mean difference was adjusted for the same confounders as were included in the analysis model. Although both physical activity variables showed some skewness, these variables were not log transformed to facilitate comparisons with our previously reported analyses. ${ }^{202936}$ All analyses were performed using Stata V.14 (Stata 2015. Stata Statistical Software).

\section{RESULTS}

\section{Physical activity at age 11 years and CDF at age 13 years}

Data to define CDF at age 13 years were more likely to be missing for children born into lower socioeconomic status households and whose mothers were more likely to experience depression and anxiety (online supplementary table S1). Physical activity data at age 11 years were available for $63.6 \%(42 / 66)$ and $67.7 \%$ (4504/6654) of children who did or did not have CDF at age 13 years, respectively.

Median levels of total and moderate-to-vigorous physical activity were lower at age 11 years in children who had CDF at age 13 years (table 1). Each $100 \mathrm{cpm}$ increase in mean total physical activity was associated with $25 \%$ lower odds of CDF at age 13 years $(\mathrm{OR}=0.75(95 \% \mathrm{CI} 0.59$ to 0.95$))$, a 1 -percentage point increase (corresponding to approximately $6 \mathrm{~min}$ ) in the daily proportion of physical activity rated as moderate to vigorous was associated with $16 \%$ lower odds of $\mathrm{CDF}(\mathrm{OR}=0.84(95 \%$

Table 2 Associations of physical activity at age 11 years with chronic disabling fatigue (CDF) at age 13 years

\begin{tabular}{|c|c|c|c|}
\hline & & Raw data $(n=4546)$ & Imputed data $(n=13978)^{*}$ \\
\hline \multirow[t]{3}{*}{ Total physical activity $\dagger$} & Unadjusted OR & $0.70(0.56,0.88)$ & $0.76(0.62,0.93)$ \\
\hline & Partially adjusted $\mathrm{OR} \ddagger$ & $0.68(0.54,0.87)$ & $0.75(0.60,0.93)$ \\
\hline & \multicolumn{2}{|c|}{ Fully adjusted mean difference (counts/min)§ } & $-77.4(-136.6,-18.1)$ \\
\hline \multirow[t]{2}{*}{ Moderate-to-vigorous physical activity } & Unadjusted OR & $0.79(0.64,0.97)$ & $0.84(0.72,0.99)$ \\
\hline & Partially adjusted OR $\ddagger$ & $0.77(0.62,0.96)$ & $0.84(0.70,0.99)$ \\
\hline \multirow[t]{4}{*}{ Sedentary time** } & Unadjusted OR & $1.50(1.15,1.95)$ & $1.38(1.07,1.79)$ \\
\hline & Partially adjusted $O R \ddagger$ & $1.50(1.15,1.95)$ & $1.39(1.07,1.80)$ \\
\hline & Fully adjusted OR§ & - & $1.35(1.02,1.79)$ \\
\hline & \multicolumn{2}{|c|}{ Fully adjusted mean difference (min/day)§ } & $26.3(2.03,50.7)$ \\
\hline
\end{tabular}

*Variables for the multiple imputation (50 imputations) were as follows: CDF at 13 and 16 years; sex; nighttime sleep duration at 7 and 9 years; body mass index (BMI) at 7 and 9 years; child mood at 9 years (Short Moods and Feelings Questionnaire score); maternal depression at 6 years (Edinburgh Postnatal Depression Scale); maternal anxiety at 6 years (Crown-Crisp Experiential Index); mean test score for English, Mathematics and Science at 11 years (Key Stage 2 tests); maternal life events score (antenatal); self-esteem at 8 years (Global Self Worth subscale from Harter's Self Perception Profile for Children); Avon Longitudinal Study of Parents and Children family adversity index (antenatal); family adversity index at 8-10 years; life difficulties, hyperactivity and internalising behaviour at 11 years (Strengths and Difficulties Questionnaire); screen time at 6 years; special school arrangements for emotional/behavioural problems at 7 years; conduct problems at 8 years; duration of breastfeeding; family income at 4 years; maternal childhood socioeconomic status; maternal education; maternal age at birth of child; maternal psychopathology at 8-10 years; experienced bullying at 8 years; days spent outdoors at 8 years; poor concentration at school at 7 years; maternal and paternal BMI at 8 years; internalising behaviour at 4 years (Strengths and Difficulties Questionnaire); early puberty; dietary patterns at 3 and 10 years.

†For total physical activity, change is per 100 counts/min increase in mean total physical activity for the whole week. $¥$ Adjusted for sex and family adversity index (antenatal).

§Adjusted for sex; family adversity index (antenatal); BMI at 7 and 9 years; maternal depression at 6 years; maternal anxiety at 6 years; internalising behaviour at 4 and 11 years; self-esteem at 8 years; child mood at 9 years; early puberty; Key Stage 2 test scores; duration of breastfeeding; family income at 4 years; screen time at 6 years; maternal childhood socioeconomic status; maternal life events score (antenatal); nighttime sleep duration at 8 years; conduct problems at 8 years; dietary patterns at 10 years. IFor moderate-to-vigorous physical activity, change is per 15 min increase (threshold $\geq 3500$ counts/min).

${ }^{* *}$ For sedentary time, change is per hour increase. 
Table 3 Associations of physical activity at age 11 years with chronic disabling fatigue (CDF) at age 16 years

\begin{tabular}{|c|c|c|c|}
\hline & & Raw data $(n=3907)$ & Imputed data $(\mathrm{n}=13978)^{*}$ \\
\hline \multirow[t]{3}{*}{ Total physical activity $\dagger$} & Unadjusted OR & $0.87(0.72,1.04)$ & $0.88(0.74,1.05)$ \\
\hline & Partially adjusted OR & $0.91(0.76,1.10)$ & $0.91(0.76,1.09)$ \\
\hline & \multicolumn{2}{|l|}{ Fully adjusted mean difference (counts/min)§ } & $-14.7(-70.8,41.4)$ \\
\hline \multirow[t]{2}{*}{ Moderate-to-vigorous physical activity? } & Unadjusted OR & $0.89(0.76,1.06)$ & $0.90(0.78,1.04)$ \\
\hline & Partially adjusted OR $¥$ & $0.95(0.80,1.13)$ & $0.94(0.81,1.09)$ \\
\hline \multirow[t]{4}{*}{ Sedentary time ${ }^{* *}$} & Unadjusted OR & $1.20(0.94,1.52)$ & $1.23(0.97,1.56)$ \\
\hline & Partially adjusted OR & $1.17(0.92,1.49)$ & $1.22(0.96,1.54)$ \\
\hline & Fully adjusted OR§ & - & $1.16(0.89,1.51)$ \\
\hline & \multicolumn{2}{|l|}{ Fully adjusted mean difference $(\mathrm{min} /$ day $) \S$} & $12.6(-9.76,35.0)$ \\
\hline
\end{tabular}

*Variables for the multiple imputation (50 imputations) were as follows: CDF at 13 and 16 years; sex; nighttime sleep duration at 7 and 9 years; body mass index (BMI) at 7 and 9 years; child mood at 9 years (Short Moods and Feelings Questionnaire score); maternal depression at 6 years (Edinburgh Postnatal Depression Scale); maternal anxiety at 6 years (Crown-Crisp Experiential Index); mean test score for English, Mathematics and Science at 11 years (Key Stage 2 tests); maternal life events score (antenatal); self-esteem at 8 years (Global Self Worth subscale from Harter's Self Perception Profile for Children); Avon Longitudinal Study of Parents and Children family adversity index (antenatal); family adversity index at 8-10 years; life difficulties, hyperactivity and internalising behaviour at 11 years (Strengths and Difficulties Questionnaire); screen time at 6 years; special school arrangements for emotional/behavioural problems at 7 years; conduct problems at 8 years; duration of breastfeeding; family income at 4 years; maternal childhood socioeconomic status; maternal education; maternal age at birth of child; maternal psychopathology at 8-10 years; experienced bullying at 8 years; days spent outdoors at 8 years; poor concentration at school at 7 years; maternal and paternal BMI at 8 years; internalising behaviour at 4 years (Strengths and Difficulties Questionnaire); early puberty; dietary patterns at 3 and 10 years.

tFor total physical activity, change is per 100 counts/min increase in mean total physical activity for the whole week

¥Adjusted for sex and family adversity index (antenatal).

$\S$ Adjusted for sex; family adversity index (antenatal); BMI at 7 and 9 years; maternal depression at 6 years; maternal anxiety at 6 years; internalising behaviour at 4 and 11 years; self-esteem at 8 years; child mood at 9 years; early puberty; Key Stage 2 test scores; duration of breastfeeding; family income at 4 years; screen time at 6 years; maternal childhood socioeconomic status; maternal life events score (antenatal); nighttime sleep duration at 8 years; conduct problems at 8 years; dietary patterns at 10 years. १For moderate-to-vigorous physical activity, change is per 15 min increase (threshold $\geq 3500$ counts/min).

${ }^{* *}$ For sedentary time, change is per hour increase.

CI 0.69 to 1.01$)$ ) and an increase of 1 hour per day in sedentary time was associated with $35 \%$ higher odds of CDF (OR $=1.35$ (95\% CI 1.02 to 1.79)) (table 2). Children with CDF at age 13 years had lower total $(-77.4$ (95\% CI -136.6 to -18.1$)$ cpm) and moderate-to-vigorous physical activity ( $-4.83(95 \%$ $\mathrm{CI}-9.63$ to -0.03$) \mathrm{min} /$ day $)$, and longer sedentary time $(26.3$ (95\% CI 2.03 to 50.7$) \mathrm{min} /$ day) at age 11 years compared with children did not develop CDF (imputed and fully adjusted mean differences).

Disabling fatigue of $3-5$ months' duration at age 13 years was less strongly associated with total physical activity (raw data unadjusted $\mathrm{OR}=0.88$ (95\% CI 0.76 to 1.03$)$; imputed data fully adjusted $\mathrm{OR}=0.86$ (95\% CI 0.75 to 0.99$)$ ), moderate-to-vigorous physical activity (raw data unadjusted $\mathrm{OR}=0.94$ (95\% CI 0.82 to 1.07 ); imputed data fully adjusted $\mathrm{OR}=0.90$ (95\% CI 0.80 to 1.01)) and sedentary time (raw data unadjusted $\mathrm{OR}=1.22(95 \%$ CI 0.99 , to 1.51$)$; imputed data fully adjusted $\mathrm{OR}=1.20(95 \%$ CI 1.00 to 1.44$)$ ).

\section{Physical activity at age 11 years and CDF at age 16 years}

Data to define CDF at age 16 years were more likely to be missing for children born into lower socioeconomic status households and whose mothers were more likely to experience depression and anxiety (online supplementary table S2). Physical activity data at age 11 years were available for $72.6 \%(53 / 73)$ and $67.8 \%(3854 / 5683)$ of children who did or did not have $\mathrm{CDF}$ at age 16 years, respectively. Median levels of total and moderate-to-vigorous physical activity were lower at age 11 years in children who had CDF at age 16 years, but with weak evidence for these differences (table 1 ). Total physical activity $(\mathrm{OR}=0.94$ (95\% CI 0.77 to 1.15$))$, moderate-to-vigorous physical activity $(\mathrm{OR}=0.96(95 \%$ CI 0.82 to 1.13$))$ and sedentary time $(\mathrm{OR}=1.16$ (95\% CI 0.89 to 1.51$))$ were not associated with $\mathrm{CDF}$ at age 16 years (table 3 ).

\section{DISCUSSION}

Children who had CDF at age 13 years had lower levels of physical activity at age 11 years. For each additional $100 \mathrm{cpm}$ of total physical activity, the odds of CDF at age 13 years were reduced by $25 \%$. Given that children with CDF in this cohort had a median physical activity level of $482 \mathrm{cpm}$, an additional $100 \mathrm{cpm}$ represents a $20 \%$ increase in the overall amount of time spent moving around. For each additional 1\% of monitored time spent in moderate-to-vigorous activity, the odds of CDF were reduced by $16 \%$. A percentage point of moderate-to-vigorous physical activity represents approximately $8 \mathrm{~min}$ of an activity such as walking or playing football (based on the average accelerometer wear time of $774 \mathrm{~min}$, hence $1 \%$ of $774=7.7 \mathrm{~min}$ ). Each additional hour of sedentary time per day was associated with $35 \%$ higher odds of CDF. Disabling fatigue of only 3-5 months' duration at age 13 years had weaker associations with physical activity, and CDF at age 16 years was not associated with physical activity at age 11 years. This suggests that the association of physical activity at age 11 years with CDF (of $>6$ months' duration) at age 13 years may reflect reverse causality, that is, the lower levels of physical activity at age 11 years are a consequence of chronic fatigue which is already present or developing and which persists until the child is 13 years old.

\section{Strengths and limitations}

Our study is the first to investigate a temporal relationship between levels of physical activity and chronic disabling fatigue (as a proxy for CFS/ME) in early and late adolescence. The main 
strengths of our study are that it used prospectively collected data from a large population based birth cohort, and that we used objective measures of physical activity. The use of DAGs enabled us to examine temporal relationships and to adjust for multiple potential confounders in a systematic way which takes account of the previous evidence in the field. ${ }^{30}$ Furthermore, we used multiple imputation to adjust for higher losses to follow-up that occur among cohort participants from less affluent social groups. ${ }^{21}$

The main limitation of our study is that children were not assessed by a doctor, which is why we describe our outcome as 'chronic disabling fatigue', a proxy for CFS/ME. At age 13 years, our classification was based only on parental report of fatigue, with limited data on degree of disability. ${ }^{6}$ We may have excluded teenagers whose parents under-reported the impact of fatigue and/or included teenagers whose parents overestimated fatigue or its impact. However, our CDF outcome at age 16 years combined parental data with a child-completed Chalder Fatigue Questionnaire (CFQ) score. ${ }^{7}$ At this age, we classified children as not having CDF if they had a CFQ score $<19$, a threshold which has high sensitivity and specificity for CFS/ME in adults. ${ }^{23}$ The threshold used to define minutes of moderate-to-vigorous physical activity from the accelerometer data affects estimates of physical activity. To facilitate comparisons with the other ALSPAC papers, we have used the threshold of $3500 \mathrm{cpm} .{ }^{25}$ We recognise that others have suggested that the Evenson et $a l^{37}$ cut-point of $\geq 2296 \mathrm{cpm}$ may have wider validity and reliability in the target group, particularly for population-based samples. ${ }^{38}$

We cannot be sure to what extent the association of physical activity at age 11 years with CDF at age 13 years reflects reverse causation. In a UK paediatric CFS/ME clinical cohort, the median (IQR) self-reported duration of illness for children aged under 12 years was $12(7-23)$ months and in children aged $12-18$ years it was 18 (11-28) months. ${ }^{3}$ Furthermore, our previous analysis of CDF among children in the ALSPAC cohort at age 13,16 and 18 years showed that $75 \%$ of children recovered after 2-3 years. ${ }^{10}$ These two studies suggest that the proportion of children in this study whose chronic fatigue would be longer than 2 years' duration would be low, and that any association of physical activity at age 11 years with CDF at age 13 years would not reflect reverse causation. However, analysis of electronic primary care data in the UK showed that children with CFS/ME had substantially higher healthcare needs than matched population controls for at least 10 years before their diagnosis. ${ }^{39}$ This suggests that parents have underestimated the duration of their child's fatigue, or that paediatric CFS/ME begins in a mild form which develops into more severe CFS/ME. This is plausible if the milder form of CFS/ME does not lead to notable absences from school or college.

\section{Our findings in the context of the studies}

Our results are not consistent with the only other study to investigate a possible association between physical activity and CFS/ME in adolescents (age 12-18 years), which found no differences in levels of physical activity 12 months before infectious mononucleosis comparing those who did or did not subsequently develop CFS/ME. ${ }^{19}$ However, this was based on self-report and a small sample (39 cases and 39 controls), and there was tentative evidence that adolescents who developed $\mathrm{CFS} / \mathrm{ME}$ had lower scores for vigorous exercise $(\mathrm{P}=0.09)$ and were spending more time napping $(\mathrm{P}=0.08)$ in the year before infectious mononucleosis. Our results are consistent with a population-based study which reported $60 \%$ higher odds $(\mathrm{OR}=1.6$
(95\% CI 1.1 to 2.3)) of 'severe fatigue' in adolescents age 13-16 years among those who had reported $>4$ hours per day of sedentary time (compared with $<2$ hours/day) 2 years previously. ${ }^{40}$ In contrast with our findings, they also reported an increased risk (50\% higher odds, $\mathrm{OR}=1.5$ (1.2 to 2.1$)$ ) associated with being physically active (exercising $>2$ times each week for an hour or more). The exposure and outcome measures in this study were self-reported (WHO Health Behaviour in School-aged Children questionnaire), and 'severe fatigue' was defined as extreme tiredness occurring more than once per week (in the last month). Crucially, this definition does not exclude tiredness caused by playing sport, which might explain the positive association between physical activity and risk of severe fatigue.

\section{CONCLUSION}

Our results suggest a possible association between lower levels of physical activity and the development of chronic fatigue in younger teenagers, but this could be explained by reverse causation. Our findings need to be replicated in a larger sample using accelerometer and fatigue data collected at repeated time points further back in time from the outcome, such that the true relationship between physical activity levels and onset of chronic fatigue can be determined.

Acknowledgements We are extremely grateful to all the families who took part in this study, the midwives for their help in recruiting them and the whole ALSPAC team, which includes interviewers, computer and laboratory technicians, clerical workers, research scientists, volunteers, managers, receptionists and nurses. The UK Medical Research Council and Wellcome (Grant ref: 102215/2/13/2) and the University of Bristol provide core support for ALSPAC. This publication is the work of the authors and EC and SMC will serve as guarantors for the contents of this paper.

Contributors SMC and TN analysed the data, wrote the first draft, revised the manuscript and approved the final version. KCD provided methodological input, analysed the data, revised the manuscript and approved the final version. RJ and ARN provided methodological input, interpreted the results, revised the manuscript and approved the final version. EC conceived the study, interpreted the results, revised the manuscript and approved the final version.

Funding This research was specifically funded by the UK MRC (Grant ref: MR/K020269/1). SMC, TN and EC were funded by an MRC research grant (MR/K020269/1); EC is also funded by an NIHR Senior Research Fellowship (SRF820138068013).

Competing interests EC is a medical advisor for the Sussex \& Kent ME/CFS Society. The other authors have indicated no potential conflicts of interest to declare.

Ethics approval Ethical approval for the study was obtained from the ALSPAC Ethics and Law Committee (IRB00003312) and the Local Research Ethics Committees.

Provenance and peer review Not commissioned; externally peer reviewed.

Data sharing statement The ALSPAC study website contains details of all the data that are available through a fully searchable data dictionary (www.bris.ac.uk/ alspac/researchers/data-access/data-dictionary/).

Open Access This is an Open Access article distributed in accordance with the terms of the Creative Commons Attribution (CC BY 4.0) license, which permits others to distribute, remix, adapt and build upon this work, for commercial use, provided the original work is properly cited. See: http://creativecommons.org/licenses/by/4.0/

(c) Article author(s) (or their employer(s) unless otherwise stated in the text of the article) 2018. All rights reserved. No commercial use is permitted unless otherwise expressly granted.

\section{REFERENCES}

1 Institute of Medicine Committee on the Diagnostic Criteria for Myalgic Encephalomyelitis/Chronic Fatigue Syndrome. Beyond myalgic encephalomyelitis/ chronic fatigue syndrome: redefining an illness. Washington (DC): National Academies Press (US), 2015

2 Bakken IJ, Tveito K, Gunnes N, et al. Two age peaks in the incidence of chronic fatigue syndrome/myalgic encephalomyelitis: a population-based registry study from Norway 2008-2012. BMC Med 2014;12:167. 
3 Collin SM, Nuevo R, van de Putte EM, et al. Chronic fatigue syndrome (CFS) or myalgic encephalomyelitis (ME) is different in children compared to in adults: a study of UK and Dutch clinical cohorts. BMJ Open 2015;5:e008830.

4 National Institute of Health and Care Excellence (NICE). Chronic fatigue syndrome/ myalgic encephalomyelitis (or encephalopathy): diagnosis and management of CFS/ ME in adults and children (NICE clinical guidelines CG53). London (UK), 2007.

5 Fukuda K, Straus SE, Hickie I, et al. The chronic fatigue syndrome: a comprehensive approach to its definition and study. International Chronic Fatigue Syndrome Study Group. Ann Intern Med 1994;121:953-9.

6 Crawley E, Hughes R, Northstone K, et al. Chronic disabling fatigue at age 13 and association with family adversity. Pediatrics 2012;130:e71-9.

7 Collin SM, Norris T, Nuevo R, et al. Chronic fatigue syndrome at age 16 years. Pediatrics 2016;137:e20153434

8 Farmer A, Fowler T, Scourfield J, et al. Prevalence of chronic disabling fatigue in children and adolescents. Br J Psychiatry 2004;184:477-81.

9 Lamers F, Hickie I, Merikangas KR. Prevalence and correlates of prolonged fatigue in a U.S. sample of adolescents. Am J Psychiatry 2013;170:502-10.

10 Norris T, Collin SM, Tilling K, et al. Natural course of chronic fatigue syndrome/myalgic encephalomyelitis in adolescents. Arch Dis Child 2017:102:522-8.

11 Jones JF, Nisenbaum R, Solomon L, et al. Chronic fatigue syndrome and other fatiguing illnesses in adolescents: a population-based study. J Adolesc Health 2004;35:34-40.

12 Chalder T, Goodman R, Wessely S, et al. Epidemiology of chronic fatigue syndrome and self reported myalgic encephalomyelitis in 5-15 year olds: cross sectional study. BMJ 2003;327:654-5.

13 Nijhof SL, Maijer K, Bleijenberg G, et al. Adolescent chronic fatigue syndrome: prevalence, incidence, and morbidity. Pediatrics 2011;127:e1169-75.

14 Rimes KA, Goodman R, Hotopf M, et al. Incidence, prognosis, and risk factors for fatigue and chronic fatigue syndrome in adolescents: a prospective community study. Pediatrics 2007;119:e603-e9.

15 Bayliss K, Goodall M, Chisholm A, et al. Overcoming the barriers to the diagnosis and management of chronic fatigue syndrome/ME in primary care: a meta synthesis of qualitative studies. BMC Fam Pract 2014;15:44.

16 Crawley EM, Emond AM, Sterne JA. Unidentified Chronic Fatigue Syndrome/myalgic encephalomyelitis (CFS/ME) is a major cause of school absence: surveillance outcomes from school-based clinics. BMJ Open 2011;1:e000252.

17 Viner R, Hotopf M. Childhood predictors of self reported chronic fatigue syndrome/ myalgic encephalomyelitis in adults: national birth cohort study. BMJ 2004;329:941.

18 ter Wolbeek M, van Doornen LJ, Kavelaars A, et al. Fatigue, depressive symptoms, and anxiety from adolescence up to young adulthood: a longitudinal study. Brain Behav Immun 2011;25:1249-55.

19 Huang Y, Katz BZ, Mears C, et al. Postinfectious fatigue in adolescents and physical activity. Arch Pediatr Adolesc Med 2010;164:803-9.

20 Riddoch CJ, Mattocks C, Deere K, et al. Objective measurement of levels and patterns of physical activity. Arch Dis Child 2007;92:963-9.
21 Boyd A, Golding J, Macleod J, et al. Cohort Profile: the 'children of the 90s'-the index offspring of the Avon Longitudinal Study of Parents and Children. Int J Epidemiol 2013;42:111-27.

22 Chalder T, Berelowitz G, Pawlikowska T, et al. Development of a fatigue scale. J Psychosom Res 1993;37:147-53.

23 Cella M, Chalder T. Measuring fatigue in clinical and community settings. J Psychosom Res 2010;69:17-22.

24 Ekelund U, Sjöström M, Yngve A, et al. Physical activity assessed by activity monitor and doubly labeled water in children. Med Sci Sports Exerc 2001;33:275-81.

25 Mattocks C, Leary S, Ness A, et al. Calibration of an accelerometer during free-living activities in children. Int J Pediatr Obes 2007;2:218-26.

26 Melanson EL, Freedson PS. Validity of the Computer Science and Applications, Inc. (CSA) activity monitor. Med Sci Sports Exerc 1995;27:934-40.

27 Mattocks C, Ness A, Leary S, et al. Use of accelerometers in a large field-based study of children: protocols, design issues, and effects on precision. J Phys Act Health 2008;5(Suppl 1):S98-111

28 Collin SM, Tilling K, Joinson C, et al. Maternal and childhood psychological factors predict chronic disabling fatigue at age 13 years. J Adolesc Health 2015;56:181-7.

29 Mattocks C, Ness A, Deere K, et al. Early life determinants of physical activity in 11 to 12 year olds: cohort study. BMJ 2008;336:26-9.

30 Moodie EE, Stephens DA. Using Directed Acyclic Graphs to detect limitations of traditional regression in longitudinal studies. Int J Public Health 2010;55:701-3.

31 Bodnar LM, Nelson MC. Maternal nutrition and fetal growth: bias introduced because of an inappropriate statistical modeling strategy may explain null findings. Am J Clin Nutr 2004;80:525-6. author reply 526-527.

32 Greenland S, Pearl J, Robins JM. Causal diagrams for epidemiologic research. Epidemiology 1999;10:37-48.

33 Textor J, Hardt J, Knüppel S. DAGitty: a graphical tool for analyzing causal diagrams. Epidemiology 2011;22:745.

34 Royston P. Multiple imputation of missing values: further update of ice, with an emphasis on interval censoring. Stata J 2007;7:445-64.

35 Rubin DB. Multiple imputation for non response in surveys. New York: Wiley and Sons, 1987.

36 Riddoch CJ, Leary SD, Ness AR, et al. Prospective associations between objective measures of physical activity and fat mass in 12-14 year old children: the Avon Longitudinal Study of Parents and Children (ALSPAC). BMJ 2009;339:b4544.

37 Evenson KR, Catellier DJ, Gill K, et al. Calibration of two objective measures of physical activity for children. J Sports Sci 2008;26:1557-65.

38 Trost SG, Loprinzi PD, Moore R, et al. Comparison of accelerometer cut points for predicting activity intensity in youth. Med Sci Sports Exerc 2011;43:1360-8.

39 Collin SM, Bakken IJ, Nazareth I, et al. Health care resource use by patients before and after a diagnosis of chronic fatigue syndrome (CFS/ME): a clinical practice research datalink study. BMC Fam Pract 2017;18:60.

40 Viner RM, Clark C, Taylor SJ, et al. Longitudinal risk factors for persistent fatigue in adolescents. Arch Pediatr Adolesc Med 2008;162:469-75. 